University of Nebraska - Lincoln

DigitalCommons@University of Nebraska - Lincoln

2002

\title{
A Multicenter, Open-Label, Randomized Comparison of Levofloxacin and Azithromycin Plus Ceftriaxone in Hospitalized Adults with Moderate to Severe Community-Acquired Pneumonia
}

\author{
Elliott Frank \\ Jersey Shore Medical Center \\ Jing Liu \\ Salem Veterans Affairs Medical Center \\ Gary Kinasewitz \\ University of Oklahoma Health Sciences Center, gary-kinasewitz@ouhsc.edu \\ Gregory J. Moran \\ Olive View-UCLA Medical Center \\ Margaret P. Oross \\ Ortho-McNeil Pharmaceutical \\ See next page for additional authors
}

Follow this and additional works at: https://digitalcommons.unl.edu/veterans

Frank, Elliott; Liu, Jing; Kinasewitz, Gary; Moran, Gregory J.; Oross, Margaret P.; Olson, William H.; Reichl, Veronica; Freitag, Susan; Bahal, Neelam; Wiesinger, Barbara A.; Tennenberg, Alan; and Kahn, James B., "A Multicenter, Open-Label, Randomized Comparison of Levofloxacin and Azithromycin Plus Ceftriaxone in Hospitalized Adults with Moderate to Severe Community-Acquired Pneumonia" (2002). U.S. Department of Veterans Affairs Staff Publications. 51.

https://digitalcommons.unl.edu/veterans/51

This Article is brought to you for free and open access by the U.S. Department of Veterans Affairs at DigitalCommons@University of Nebraska - Lincoln. It has been accepted for inclusion in U.S. Department of Veterans Affairs Staff Publications by an authorized administrator of DigitalCommons@University of Nebraska - Lincoln. 


\section{Authors}

Elliott Frank, Jing Liu, Gary Kinasewitz, Gregory J. Moran, Margaret P. Oross, William H. Olson, Veronica Reichl, Susan Freitag, Neelam Bahal, Barbara A. Wiesinger, Alan Tennenberg, and James B. Kahn 


\title{
A Multicenter, Open-Label, Randomized Comparison of Levofloxacin and Azithromycin Plus Ceftriaxone in Hospitalized Adults with Moderate to Severe Community-Acquired Pneumonia
}

\author{
Elliott Frank, MD, ${ }^{1}$ Jing Liu, $M D,{ }^{2}$ Gary Kinasewitz, $M D,{ }^{3}$ \\ Gregory J. Moran, MD, Margaret P. Oross, BSN, MHSA, \\ William H. Olson, PhD, ${ }^{5}$ Veronica Reichl, RN, ${ }^{6}$ Susan Freitag, $M S,{ }^{6}$ \\ Neelam Bahal, PharmD, Barbara A. Wiesinger, BSN, ${ }^{5}$ \\ Alan Tennenberg, MD, PhD, and James B. Kahn, $\mathrm{MD}^{5}$ \\ ${ }^{I}$ Jersey Shore Medical Center, Neptune, New Jersey, ${ }^{2}$ Salem Veterans Affairs Medical \\ Center, Salem, Virginia, ${ }^{3}$ University of Oklahoma Health Sciences Center, Oklahoma \\ City, Oklahoma, ${ }^{4}$ Olive View-UCLA Medical Center, Sylmar, California, ${ }^{5}$ Ortho-McNeil \\ Pharmaceutical, Raritan, New Jersey, and ${ }^{6}$ Advanced Biologics LLC, Lambertville, \\ New Jersey
}

\begin{abstract}
Background: Changing etiologic patterns and the growing problem of antimicrobial resistance, particularly an increase in macrolide-resistant pneumococcal bacteremia, are causing physicians to adopt new approaches to the treatment of community-acquired pneumonia (CAP).

Objective: The relative efficacy and tolerability of levofloxacin monotherapy and azithromycin and ceftriaxone combination therapy were assessed in hospitalized adults with moderate to severe CAP.

Methods: This Phase IV, multicenter, open-label, randomized trial compared 2 treatment regimens: (1) levofloxacin $500 \mathrm{mg}$ PO or IV q24h, and (2) azithromycin $500 \mathrm{mg}$ IV q24h for $\geq 2$ days plus ceftriaxone $1 \mathrm{~g}$ IV q24h for 2 days, followed by an optional transition to azithromycin $500 \mathrm{mg}$ PO q24h at the investigator's discretion. The total duration of therapy was to be a minimum of 10 days in both treatment groups. Ceftriaxone was included in the initial azithromycin regimen to ensure coverage against pneumococcal bacteremia.

Results: Of 236 patients in the intent-to-treat population, completion or withdrawal information was available for 110 patients in the levofloxacin group and 114 in the azithromycin group. Baseline demographic and disease characteristics were comparable between groups. At the end of treatment, the clinical success rate (cured + improved) in
\end{abstract}

These results were presented at the 37 th Annual Meeting of the Infectious Diseases Society of America, November 18-21, 1999, Philadelphia, Pennsylvania.

Accepted for publication June 25, 2002.

Printed in the USA. Reproduction in whole or part is not permitted.

This article is a U.S. government work, and is not subject to copyright in the United States. 
clinically evaluable patients was $94.1 \%$ in the levofloxacin group and $92.3 \%$ in the azithromycin group. The respective posttherapy microbiologic eradication rates were $89.5 \%$ and $92.3 \%$. Levofloxacin was as well tolerated as azithromycin, with an incidence of drug-related adverse events (AEs) for all body systems of $5.3 \%$ and $9.3 \%$, respectively. One patient receiving levofloxacin had a serious drug-related $\mathrm{AE}$, compared with 7 patients receiving azithromycin.

Conclusions: In this study in hospitalized patients with moderate to severe CAP, levofloxacin monotherapy was at least as effective as a combination regimen of azithromycin and ceftriaxone in providing coverage against the current causative pathogens in CAP. In addition, levofloxacin was as well tolerated as the combination of azithromycin and ceftriaxone.

Key words: levofloxacin, communityacquired pneumonia, fluoroquinolones, clinical trial. (Clin Ther. 2002;24:12921308)

\section{INTRODUCTION}

Despite the availability of effective antimicrobial agents, community-acquired pneumonia (CAP) remains a potentially life-threatening infection of the lower respiratory tract. The overall mortality rate among patients hospitalized with CAP ranges from $5 \%$ to $25 \%$, whereas the mortality rate in those with severe illness requiring treatment in the intensive care unit ranges from $20 \%$ to $50 \% .^{1,2}$ Streptococcus pneumoniae is the most common etiologic agent in hospitalized patients with CAP, particularly those with severe disease ${ }^{3,{ }^{4}}$ Pneumococcal bacteremia is a ma jor complication in hospitalized patients with CAP, with an attributable mortality rate ranging from $20 \%$ to $41 \% .{ }^{5}$ The mortality rate in patients with severe CAP who are bacteremic is $\sim 3$ times that in patients with severe CAP who have negative blood cultures. ${ }^{6}$

The significant risk of bacteremia in patients with moderate to severe CAP supports selection of an antibiotic regimen for initial in-hospital therapy that is active against the main causative pathogens while achieving high drug concentrations in plasma and lung tissue. Results of a retrospective investigation in patients with severe bacteremic pneumococcal pneumonia suggest that monotherapy may produce suboptimal outcomes in this population. ${ }^{7}$ However, a retrospective review of 98 cases of CAP-associated pneumococcal bacteremia from prospective, randomized, controlled clinical trials demonstrated a clinical success rate of $96.9 \%$ and a microbiologic success rate of $95.9 \%$ with levofloxacin $500 \mathrm{mg} / \mathrm{d} .{ }^{8} \mathrm{In}$ addition, the Infectious Diseases Society of America (IDSA) recommends use of doxycycline, macrolides, or fluoroquinolones for the empiric treatment of most outpatient cases of CAP and either a fluoroquinolone alone or a beta-lactam/beta-lactamase inhibitor or extended-spectrum cephalosporin combined with a macrolide for most hospitalized patients with CAP in a general medical ward. ${ }^{9}$

This study compares the efficacy and tolerability of 2 regimens-monotherapy with levofloxacin and combination therapy with azithromycin and ceftriaxonethat meet IDSA criteria for the treatment of CAP. Both are used for empiric treatment of moderate to severe CAP, although recently there has been increased concern about the association of macrolides with macrolide-resistant pneumococcal bacteremia. $^{10,11}$ 


\section{PATIENTS AND METHODS}

\section{Study Design}

This was a Phase IV, multicenter, openlabel, randomized trial comparing the efficacy and tolerability of levofloxacin monotherapy and the combination of azithromycin and ceftriaxone in hospitalized patients with moderate to severe CAP.

\section{Inclusion and Exclusion Criteria}

Patients aged $\geq 18$ years were eligible for the study if they had a diagnosis of moderate to severe pneumonia acquired in the community or in a nursing home. The diagnostic criteria included (1) characteristic clinical signs, including $\geq 1$ of the following - fever (oral temperature $>38^{\circ} \mathrm{C}$ ), hypothermia (oral temperature $<35.5^{\circ} \mathrm{C}$ ), leukocytosis $(>10,000$ white blood cells $/ \mathrm{mm}^{3}$ ), or bands $>10 \%$; (2) radiologic evidence of pneumonia (an acute infiltrate consistent with pneumonia on chest radiography); (3) collection of a mucopurulent sputum specimen for culture and Gram's staining within 24 hours before study drug administration; and (4) a Fine risk score ${ }^{12}$ of 71 to 130 (indicative of moderate to severe disease and associated need for hospitalization) at study inclusion. Patients who had received previous antimicrobial therapy for any infection were allowed to participate if the total duration of previous therapy was $\leq 24$ hours or the patient had received $>72$ hours of therapy but was classified as a treatment failure.

Women of childbearing potential were required to have had normal menstrual flow in the month before study entry, to have a negative result on serum pregnancy testing (human chorionic gonadotropin $\beta$ subunit) immediately before study entry or a negative urine pregnancy test (sensitive to $50 \mathrm{mIU} / \mathrm{mL}$ ) if serum pregnancy testing would delay treatment; and to have been using hormonal contraception for $\geq 1$ month before study entry, to have an intrauterine device, or to consent to using a spermicide/barrier method of contraception. Whichever method of contraception was in use at the time of study entry was to be continued throughout the study. Women who had been postmenopausal for $\geq 1$ year or had been rendered surgically menopausal were also eligible.

Patients were excluded from study participation if they had an infection caused by a pathogen known or suspected to be resistant to any of the study drugs before their adinission to the study or if they had experienced an allergic reaction or serious adverse reaction to levofloxacin, azithromycin, ceftriaxone, or any other member of the quinolone, macrolide, or beta-lactam class of antimicrobial agents. Patients were also ineligible if they had been hospitalized within 2 weeks before study entry (or within 1 month before study entry if antimicrobial therapy had been administered during this time), or if their life expectancy was $<72$ hours.

Other exclusion criteria included creatinine clearance $<20 \mathrm{~mL} / \mathrm{min}$ (when only serum creatinine data were available, the Cockcroft-Gault equation was used to calculate creatinine clearance); empyema or the presence of pleural fluid requiring an indwelling chest tube; pneumonia due to aspiration of gastric contents; HIV infection, with a CD4 cell count $<200 / \mathrm{cm}^{3}$; presence of any seizure disorder or a psychiatric condition requiring chronic use of tranquilizers; or presence of any disease or disorder that could interfere with evaluation of the study treatments. Patients who had received any experimental drug 
within 30 days before study entry were also excluded.

All patients, or their legally authorized representatives, provided written informed consent.

\section{Study Treatments}

Randomization was conducted according to a computer-generated schedule prepared by Ortho-McNeil before initiation of the study. Patients were randomized in a 1:1 ratio to 1 of 2 treatment groups: levofloxacin $500 \mathrm{mg}$ PO or IV q24h, or azithromycin $500 \mathrm{mg}$ IV q24h for $\geq 2$ days plus ceftriaxone $1 \mathrm{~g} \mathrm{IV}$ q24h for 2 days, followed by an optional transition to azithromycin $500 \mathrm{mg}$ PO q24h at the investigator's discretion. Ceftriaxone was included in the initial azithromycin regimen to ensure coverage against pneumococcal bacteremia. The first dose of study drug was administered as soon as admission procedures were completed and could be given in the emergency department. Thereafter, all doses were administered in the morning. Oral levofloxacin therapy could be started at the time of study entry or as soon as oral medication could be tolerated. The duration of therapy was to be a total of 10 days in both treatment groups.

Levofloxacin could be administered without regard to meals, whereas oral azithromycin was taken $\geq 1$ hour before or $>2$ hours after a meal. In the levofloxacin group, patients with a calculated creatinine clearance of 20 to $49 \mathrm{~mL} / \mathrm{min}$ received an initial loading dose of levofloxacin $500 \mathrm{mg}$, followed by $250 \mathrm{mg}$ q24h. Patients with hepatic or renal impairment were administered azithromycin and ceftriaxone with caution, as specified in the respective package inserts.
The use of systemic corticosteroids was contraindicated during the study, unless such therapy was already being received for an unrelated medical condition. Patients requiring antacid therapy were strongly advised to use compounds containing calcium carbonate rather than magnesium-aluminum hydroxide or sucralfate. If either of the latter was used, it was to be taken $>2$ hours after study drug administration. Theophylline levels were closely monitored as clinically appropriate in both treatment groups, with dose adjustment as necessary. Because concurrent use of macrolides or some quinolones and warfarin or its derivatives has been associated with increased anticoagulant effects in clinical practice, ${ }^{13}$ the prothrombin time was closely monitored as clinically indicated in patients receiving warfarin or its derivatives. Given reports of drug interactions between macrolides and digoxin, ergotamine/dihydroergotamine, triazolam, carbamazepine, terfenadine, cyclosporine, hexobarbital, and phenytoin ${ }^{14}$ patients receiving any of these drugs in combination with azithromycin were monitored closely as clinically indicated.

\section{Study Evaluations}

Patients were evaluated at the initial (admission) visit, at which time a medical history was obtained and a physical examination was performed. The severity of pneumonia was assessed in terms of the Fine risk score, ${ }^{12}$ and posteroanterior and lateral chest radiographs were obtained. All patients were required to have a mucopurulent sputum specimen, which was sent for Gram's staining and culture. $\Lambda$ urine specimen was obtained for pregnancy testing, if applicable. Blood sam- 
ples were obtained for culture, serum pregnancy testing, complete blood count with differential, and measurement of arterial $\mathrm{pH}$, blood urea nitrogen, sodium, glucose, and arterial partial pressure of oxygen. Any concomitant therapies were recorded. Treatment with study drug was then initiated.

Patients' clinical status was evaluated $\sim 72$ hours after the start of treatment (days 3-5). Those whose condition had not stabilized or whose clinical signs/symptoms had worsened after 72 hours were withdrawn from the study and were considered treatment failures. These patients were switched to an appropriate alternative therapy. Those whose condition had stabilized but was not yet improved could continue in the study at the investigator's discretion. Patients whose admission organism was determined to be resistant to study drug could also remain in the study at the investigator's discretion, provided they were clinically stable or improving.

General, nondirected questioning was used to elicit adverse events (AEs) occurring during treatment. Blinding to treatment assignment was not possible because of the investigator's freedom to change the route of administration or discontinue ceftriaxone.

Between days 2 and 7 after the completion of therapy (or at early withdrawal), a physical examination was performed, including recording of vital signs. Clinical signs and symptoms were evaluated, and the patient's clinical response was assessed. Posteroanterior and lateral chest radiographs were obtained, as was a sputum sample for Gram's staining and culture, if available. If the admission culture had been positive or the patient was considered a clinical failure, a blood sample was drawn for routine culture. Patients were questioned about any AEs that had occurred during the study.

\section{Efficacy and Safety Assessments}

The primary efficacy variable was the clinical response at the posttherapy (or early-withdrawal) assessment in clinically evaluable patients (see Statistical Analysis section for definitions of study populations). Secondary efficacy variables included the posttherapy microbiologic response by infection; the posttherapy microbiologic response for each pathogen isolated from an admission sputum culture; and the posttherapy microbiologic response for blood pathogens isolated at admission.

\section{Clinical Efficacy}

Clinical efficacy was assessed by comparing the signs, symptoms, and radiologic findings from the posttherapy (or earlywithdrawal) evaluation with those from the admission evaluation. The following definitions were used to categorize the clinical response: clinical cure-resolution of pretreatment clinical signs and symptoms (radiographic resolution not required) and no need for additional antimicrobial therapy; improved-significant improvement in clinical findings, but clinical evidence of resolution incomplete during therapy or at follow-up evaluation in a patient not requiring additional antimicrobial therapy; failure--incomplete response or no apparent response to therapy, and need for additional antimicrobial therapy; and unevaluable - clinical evaluation of cure, improvement, or failure not possible because of inadequate follow-up data, use of concomitant antimicrobial therapy, or protocol violation. 


\section{E. FRANK ET AL.}

\section{Microbiologic Efficacy}

Microbiologic response was assessed by comparing the results of culture from the posttherapy (or early-withdrawal) evaluation with those from the admission evaluation. Based on the posttherapy (or early-withdrawal) culture results, each admission pathogen was assigned to 1 of the following categories of microbiologic response: eradicated-negative results on culture in the absence of additional antimicrobial therapy; presumed eradicated - no respiratory specimen available for culture and a clinical response of clinical cure or improved in the absence of additional antimicrobial therapy; persisted-positive posttherapy culture; presumed persisted-in patients with a clinical response of clinical failure, no culture or a negative test-of-cure culture while receiving additional antimicrobial therapy; and unable to evaluate - in patients with a clinical response of clinical cure, improved, or unable to evaluate, no test-ofcure culture due to loss to follow-up or premature withdrawal, or a negative culture or no test-of-cure culture in the presence of additional antimicrobial therapy. Later reevaluation identified any organisms that had been susceptible to study treatment at admission but subsequently acquired resistance.

At the posttherapy evaluation, each paticnt's infection was assigned to 1 of the following categories of microbiologic response based on the microbiologic response of all pathogens isolated in that patient at admission: eradicated/presumed eradicated-all pathogens isolated at admission were eradicated or presumed to have been eradicated; mixed $-\geq 1$ pathogen isolated at admission was eradicated or presumed to have been eradicated, while others persisted or were presumed to have persisted; persisted/presumed persistedall pathogens isolated at admission persisted or were presumed to have persisted; and unable to evaluate - a judgment could not be made.

Bacteremia was defined as 1 blood culture result positive for a pathogen at admission. In patients for whom no blood culture was available at the posttherapy evaluation, the microbiologic response was classified as unknown if the clinical response was cured or improved and as presumed persisted if the clinical response was failure. Superinfection was defined as the emergence or worsening of clinical signs and symptoms requiring additional antimicrobial therapy when a pathogen not isolated at admission was isolated from any site through the posttherapy evaluation.

\section{Safety}

Tolerability was assessed based on findings of the physical examinations and laboratory tests performed during the study and AEs reported by patients. Safety parameters were assessed separately for oral and parenteral therapy. Treatmentemergent AEs were defined as new-onset AEs or existing AEs whose severity or frequency was exacerbated after administration of study drug, based on the investigator's judgment.

\section{Analysis Plan}

Five populations were identified for the purposes of analysis. The intent-to-treat population included all randomized patients. The modified intent-to-treat population included those patients who had a confirmed diagnosis of moderate to severe CAP and received $\geq 1$ dose of study drug. The clinically evaluable population 
included patients who had clinical signs/ symptoms at admission and a chest radiograph consistent with acute pneumonia; had a clinical response other than unable to evaluate; had received consecutive doses of protocol-specified drug on days 1 through 3 at minimum and $\geq 7$ doses during the first 10 days (patients who discontinued study treatment as a result of clinical failure after 72 hours of consecutive therapy were considered evaluable); had received no concomitant or posttherapy treatment with another effective systemic antibiotic; and had a valid posttherapy evaluation. The microbiologically evaluable population included patients who were evaluable for clinical efficacy; had a specimen for culture obtained within 24 hours before starting study treatment; had an admission pathogen recognized as a potential cause of CAP; and had a valid posttherapy evaluation, including a specimen for culture, if available. The safety population included patients who took $\geq 1$ dose of study medication.

The primary efficacy variable was the posttherapy clinical response (cured, improved, failed, or unable to evaluate) in clinically evaluable patients. Clinical success was derived by combining the clinical responses cured and improved. Clinical success rates and by-patient microbiologic eradication rates were analyzed by treatment group for each population.

To evaluate whether levofloxacin was at least as effective as azithromycin, 2-sided 95\% Cls (normal approximation to the binomial with a continuity correction) were computed around the treatment differences (azithromycin levofloxacin) in posttherapy response rates. ${ }^{15}$ Statistical equivalence (ie, levofloxacin at least as effective as azithromycin) was considered to be demonstrated if the $95 \%$ CI crossed zero and the upper limit was $\leq 15 \% .^{16}$

Posttherapy microbiologic eradication rates were summarized for microbiologically evaluable patients in each treatment group by pathogen category (grampositive vs gram-negative aerobes); pathogen and infection overall; and pathogen within each culture category (respiratory, blood, most prevalent pathogens). Twosided $95 \%$ CIs were computed around the treatment differences (azithromycin levofloxacin) in posttherapy microbiologic response rates. In microbiologically evaluable patients, a cross-tabulation of by-patient microbiologic response versus clinical response was performed. Pathogens were classified by those isolated from respiratory cultures and those isolated from blood cultures. In clinically evaluable patients, posttherapy clinical response rates by pathogen within each culture category were summarized for the most prevalent pathogens (those isolated at admission from $\geq 5$ patients in each treatment group).

Approximately 198 patients were to be randomized to treatment to provide 148 clinically evaluable patients, with a minimum of 74 patients in each treatment group. This would provide $80 \%$ power to test the null hypothesis, assuming an $88 \%$ clinical success rate in each of the treatmont groups, at a significancc level of 0.025 .

\section{RESULTS}

Between December 15, 1997, and June 7, 1999,236 patients were randomized to treatment and received 1 of the 2 study regimens (Table I). These patients composed the intent-to-treat population. The levofloxacin group received IV treatment 
Table I. Patient disposition, intent-to-treat (ITT) population (all randomized patients).

\begin{tabular}{lcc}
\hline & \multicolumn{2}{c}{ No. (\%) } \\
\cline { 2 - 3 } & Levofloxacin & Comparator Regimen $^{*}$ \\
\hline IIT population & 115 & 121 \\
No. with completion/withdrawal information ${ }^{\dagger}$ & 110 & 114 \\
Lost to follow-up & 5 & 7 \\
Total completing study & $92(83.6)$ & $86(75.4)$ \\
Total withdrawals & $23(20.9)$ & $35(30.7)$ \\
Reason for withdrawal & & \\
Adverse event & $5(4.5)$ & $5(4.4)$ \\
Clinical failure & $4(3.6)$ & $6(5.3)$ \\
Negative radiographic findings & $3(2.7)$ & $4(3.5)$ \\
Personal reason & $2(1.8)$ & $2(1.8)$ \\
No study drug taken & $2(1.8)$ & $3(2.6)$ \\
Other & $7(6.4)$ & $15(13.2)$ \\
Modified ITT population ${ }^{\ddagger}$ & 106 & 105 \\
Clinically evaluable population & 85 & 78 \\
Microbiologically evaluable population & 36 & 35 \\
\hline
\end{tabular}

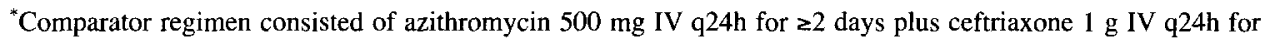
2 days, followed by an optional switch to azithromycin $500 \mathrm{mg}$ PO q24h at the investigator's discretion.

${ }^{\dagger}$ Percentages are based on number of patients with completion/withdrawal information.

${ }^{\ddagger}$ Patients in ITT population with a confirmed diagnosis of moderate to severe community-acquired pneumonia who took $\geq 1$ dose of study drug.

for a mean of 3.67 days, and the azithromycin group received IV treatment for a mean of 3.83 days (plus a mean 2.36 days of IV ceftriaxone). Five patients in the levofloxacin group and 7 in the azithromycin group were lost to follow-up (eg, were transferred to another facility). Completion/withdrawal data were therefore available for 110 patients in the levofloxacin group and 114 in the azithromycin group. Twenty-three $(20.9 \%)$ patients in the levofloxacin group withdrew prematurcly: 5 (4.5\%) duc to AEs, 4 (3.6\%) for clinical failure, $3(2.7 \%)$ due to a negative radiograph, and $11(10.0 \%)$ for per- sonal or other reasons or because no study drug was taken. Thirty-five $(30.7 \%)$ patients in the azithromycin group withdrew prematurely: $5(4.4 \%)$ due to AEs, 6 $(5.3 \%)$ for clinical failure, 4 (3.5\%) due to a negative radiograph, and $20(17.5 \%)$ for personal or other reasons or because no study drug was taken. A total of 211 patients were included in the modified intent-to-treat population, 163 of whom were clinically evaluable and 71 of whom were microbiologically evaluable.

Bascline demographic and discase characteristics were similar in clinically evaluable patients in the 2 treatment groups 
(Table II). The mean duration of pneumonia at baseline was 7.3 days, and the mean Fine risk score was 93.4. Somewhat less than one third $(47 / 163)$ of patients were current smokers, with a history of 68.9 pack years (data not shown).

Table III lists the clinical response rates at the posttherapy (or early-withdrawal) evaluation. In clinically evaluable patients, the clinical success rate was $94.1 \%$ in the levofloxacin group and $92.3 \%$ in the azithromycin group $(95 \% \mathrm{CI},-10.20$ to 6.58). The clinical success rates in the modified intent-to-treat population were a respective $89.6 \%$ and $85.7 \%(95 \% \mathrm{CI}$, -13.24 to 5.43 ). In microbiologically

Table II. Baseline demographic and disease characteristics of clinically evaluable patients.

\begin{tabular}{|c|c|c|c|}
\hline & $\begin{array}{l}\text { Levofloxacin } \\
\qquad(\mathrm{n}=85)\end{array}$ & $\begin{array}{c}\text { Comparator Regimen }{ }^{\dagger} \\
(\mathrm{n}=78)\end{array}$ & $\begin{array}{c}\text { Total } \\
(\mathrm{N}=163)\end{array}$ \\
\hline \multicolumn{4}{|l|}{ Sex, no. $(\%)$} \\
\hline Male & $56(65.9)$ & $60(76.9)$ & $116(71.2)$ \\
\hline Female & $29(34.1)$ & $18(23.1)$ & $47(28.8)$ \\
\hline \multicolumn{4}{|l|}{ Age, $y$} \\
\hline Mean \pm SD & $67.8 \pm 13.11$ & $67.3 \pm 13.17$ & $67.6 \pm 13.10$ \\
\hline Range & 29-94 & $41-90$ & 29-94 \\
\hline \multicolumn{4}{|l|}{ Race, no. (\%) } \\
\hline White & $68(80.0)$ & $56(71.8)$ & $124(76.1)$ \\
\hline Black & $8(9.4)$ & $11(14.1)$ & $19(11.7)$ \\
\hline Asian & $1(1.2)$ & $0(0.0)$ & $1(0.6)$ \\
\hline Other & $8(9.4)$ & $11(14.1)$ & $19(11.7)$ \\
\hline \multicolumn{4}{|l|}{ Body weight, lb } \\
\hline Mean \pm SD & $171.9 \pm 46.85$ & $160.6 \pm 41.31$ & $166.7 \pm 44.58$ \\
\hline Range & 95-355 & $70-273$ & $70-355$ \\
\hline Data missing, no. (\%) & $5(5.9)$ & $9(11.5)$ & $14(8.6)$ \\
\hline \multicolumn{4}{|l|}{ Duration of illness, $d$} \\
\hline Mean \pm SD & $7.3 \pm 6.26$ & $7.2 \pm 7.23$ & $7.3 \pm 6.72$ \\
\hline Range & $1-32$ & $1-47$ & $1-47$ \\
\hline Data missing, no. (\%) & $1(1.2)$ & $1(1.3)$ & $2(1.2)$ \\
\hline \multicolumn{4}{|l|}{ Fine risk score ${ }^{\ddagger}$} \\
\hline Mean \pm SD & $91.3 \pm 16.93$ & $95.8 \pm 20.24$ & $93.4 \pm 18.67$ \\
\hline Range & $61-136$ & $62-149$ & $61-149$ \\
\hline
\end{tabular}

*Patients with clinical signs/symptoms and a chest radiograph consistent with acute pneumonia at admission who had a clinical response other than unable to evaluate, had received consecutive doses of study drug on days 1 through 3 and $\geq 7$ doses in the first 10 days and no concomitant or posttherapy treatment with another systemic antibiotic, and had a valid posttherapy evaluation.

'Comparator regimen consisted of azithromycin $500 \mathrm{mg}$ IV q24h for $\geq 2$ days plus cettriaxone $1 \mathrm{~g}$ IV q24h for 2 days, followed by an optional switch to azithromycin $500 \mathrm{mg} \mathrm{PO}$ q24h at the investigator's discretion.

${ }^{\ddagger}$ A score of 71 to 130 is indicative of moderate to severe disease and associated need for hospitalization. 
Table III. Clinical response rates at the end of therapy.

\begin{tabular}{lccc}
\hline & \multicolumn{2}{c}{ Clinical Success, ${ }^{*}$ no./N (\%) } & \\
\cline { 2 - 3 } Subgroup & Levofloxacin & Comparator Regimen & \\
\hline Intent-to-treat $^{\S}$ & $100 / 115(87.0)$ & $97 / 121(80.2)$ & -16.63 to 3.04 \\
Modified intent-to-treat" $_{\text {Clinically evaluable }}$ & $95 / 106(89.6)$ & $90 / 105(85.7)$ & -13.24 to 5.43 \\
Microbiologically evaluable & $80 / 85(94.1)$ & $72 / 78(92.3)$ & -10.20 to 6.58 \\
& $33 / 36(91.7)$ & $33 / 35(94.3)$ & -10.67 to 15.91 \\
\hline
\end{tabular}

${ }^{*}$ Clinical success $=$ cure + improved.

${ }^{\dagger}$ Comparalor regimen consisted of acithromycin 500 ng IV y24h for $\geq 2$ days plus ceftriaxone 1 g IV y24h for 2 days, followed by an optional switch to azithromycin $500 \mathrm{mg}$ PO q24h at the investigator's discretion.

${ }^{\ddagger}$ Two-sided $95 \% \mathrm{CI}$ around the difference (comparator regimen - levofloxacin) in clinical success rates.

${ }^{8}$ Five patients in the levofloxacin group and 10 patients in the comparator group were considered clinical successes based solely on the evaluation conducted during therapy.

"Four patients in the levofloxacin group and 2 patients in the comparator group were considered clinical successes based solely on the evaluation conducted during therapy.

evaluable patients, the clinical success rates were $91.7 \%$ and $94.3 \%$, respectively (95\% CI, -10.67 to 15.91$)$. Twelve patients in the levofloxacin group received only oral levofloxacin therapy. The clinical success rate in these patients was $100 \%$.

Clinical and microbiologic response rates were comparable within each treatment group (Table IV). All 33 microbiologically evaluable patients in the levofloxacin group who had a posttherapy microbiologic response of eradicated had a clinical responsc of cure or improved. The 3 patients with a microbiologic response of persisted were classified as clinical failures. Of 35 patients in the azithromycin group who were microbiologically evaluable and had a posttherapy microbiologic response of eradicated, $33(94.3 \%)$ had a clinical response of cure or improved. The remaining 2 $(5.7 \%)$ patients were considered clinical failures.
$S$ pneumoniae was isolated from sputum cultures obtained from 29 patients, 14 in the levofloxacin group and 15 in the azithromycin group. Haemophilus influenzae was isolated from sputum cultures obtained from 22 patients, 9 in the levofloxacin group and 13 in the azithromycin group. The eradication rate for $S$ pneumoniae in sputum cultures was $100 \%$ in both the levofloxacin and azithromycin groups (95\% CI, -3.57 to 3.57 ); the eradication rate for $H$ influenzae was also $100 \%$ in both groups (95\% CI, -5.56 to 5.56 ). $S$ pneumoniae was also isolated from blood cultures obtained from 5 patients in the clinically evaluable population, 3 in the levofloxacin group and 2 in the azithromycin group. The eradication rate for $S$ pneumoniae in blood cultures was $100 \%$ in both the levofloxacin and azithromycin groups. All other pathogens isolated from respiratory or blood cultures occurred in $<5$ patients in either treatment group. 
Table IV. Comparison of microbiologic and clinical response at the end of therapy.

A. Levofloxacin $(n=36)$

Clinical Response, No. (\%)

Cure Improved Failure Unable to Evaluate Total

Microbiologic response

Eradicated*

Persisted $^{\dagger}$

Total

$29(87.9) \quad 4(12.1) \quad 0(0)$

$0(0)$

$0(0)$

33

$0(0) \quad 0(0) \quad 3(100)$

$29(80.6) \quad 4(11.1) \quad 3(8.3)$

$0(0)$

3

36

B. Comparator Regimen ${ }^{\ddagger}(n=35)$

Clinical Response, No. (\%)

Cure Improved Failure Unable to Evaluate Total

Microbiologic response

Eradicated $^{*}$

Persisted $^{\dagger}$

$23(65.7) \quad 10(28.6) \quad 2(5.7)$

$0(0)$

35

$0(0) \quad 0(0) \quad 0(0)$

$23(65.7) \quad 10(28.6) \quad 2(5.7)$

$0(0)$

$0(0)$

Total

$23(65.7) \quad 10(28.6)-2(5.7)$

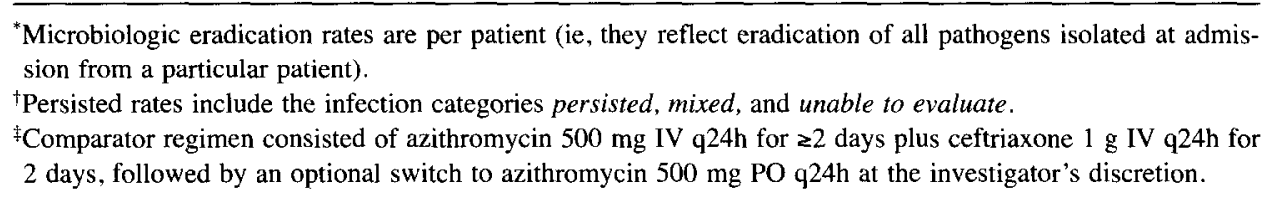

Table $\mathrm{V}$ presents the susceptibilities to the study drugs of the 122 bacterial isolates cultured at admission. The susceptibilities of 18 isolates were unknown. Of 104 admission isolates for which susceptibilities were determined, 103 were susceptible to levofloxacin, 95 to azithromycin, and 100 to ceftriaxone. One isolate was intermediately susceptible to levofloxacin, 1 was intermediately susceptible to azithromycin, and 3 were intermediately susceptible to ceftriaxone. None of the 104 isolates were resistant to levofloxacin, whereas 8 were resistant to azithromycin and 1 was resistant to ceftriaxone.

The cross-susceptibilities of admission pathogens to levofloxacin and azithromy- cin are shown in Table VI. Of 103 isolates that were susceptible to levofloxacin, 95 were also susceptible to azithromycin, 1 was intermediately susceptible, and 7 were resistant. The 1 isolate that was intermediately susceptible to levofloxacin was resistant to azithromycin. Table VII shows the cross-susceptibilities of admission pathogens to levofloxacin and ceftriaxone. Of 103 isolates that were susceptible to levofloxacin, 99 were also susceptible to ceftriaxone, 3 were intermediately susceptible, and 1 was resistant. The 1 isolate that was intermediately susceptible to levofloxacin was susceptible to ceftriaxone.

The clinical characteristics of the 5 patients classified as treatment failures are 
Table V. Susceptibility to study drugs of admission pathogens.

\begin{tabular}{lccr}
\hline & \multicolumn{3}{c}{ No. of Isolates } \\
\cline { 2 - 4 } & Levofloxacin $^{*}$ & Azithromycin & Ceftriaxone \\
\hline Susceptible & 103 & 95 & 100 \\
Intermediately susceptible & 1 & 1 & 3 \\
Resistant & 0 & 8 & 1 \\
Unknown & 18 & 18 & 18 \\
Total & 122 & 122 & 122 \\
\hline
\end{tabular}

"Two patients in the levofloxacin group and 3 patients in the comparator group were randomized to treatment but did not receive study medication.

Table VI. Cross-susceptibility to levofloxacin and azithromycin of admission pathogens.

\begin{tabular}{lccccr}
\hline & \multicolumn{5}{c}{ Azithromycin, ${ }^{*}$ No. of Isolates } \\
\cline { 2 - 6 } & Susceptible & $\begin{array}{c}\text { Intermediately } \\
\text { Susceptible }\end{array}$ & Resistant & Unknown & Total \\
\hline Levofloxacin, ${ }^{*}$ no. of isolates & 95 & 1 & 7 & 0 & 103 \\
$\quad$ Susceptible & 0 & 0 & 1 & 0 & 1 \\
Intermediately susceptible & 0 & 0 & 0 & 0 & 0 \\
Resistant & 0 & 0 & 0 & 18 & 18 \\
Unknown & 95 & 1 & 8 & 18 & 122 \\
Total & 95 & & & \\
\hline
\end{tabular}

*Two patients in the levofloxacin group and 3 patients in the comparator group were randomized to treatment but did not receive study medication.

listed in Table VIII. All patients in both treatment groups had significant comorbidity and reccived study drug for $\leq 5$ days. The 3 causative organisms in the levofloxacin group were $S$ pneumoniae (minimum inhibitory concentration [MIC] 1.0 $\mu \mathrm{g} / \mathrm{mL}$ ), Staphylococcus aureus (MIC 0.5 $\mu \mathrm{g} / \mathrm{mL}$ ), and $H$ influenzae (MIC not available). Both organisms with documented MICs were susceptible to levofloxacin. The 2 causative organisms in the azithromycin group were $H$ influenzae (MIC 0.5 $\mu \mathrm{g} / \mathrm{mL}$ ) and Escherichia coli (MIC 8.0 $\mu \mathrm{g} / \mathrm{mL})$. Because $H$ influenzae is considered susceptible to azithromycin if its MIC is $54.0 \mu \mathrm{g} / \mathrm{mL}$ and $E$ coli is considered susceptible if its $\mathrm{MIC}$ is $\leq 2 \mu \mathrm{g} / \mathrm{mL}$, the former was susceptible to azithromycin. None of the cases of pneumococcal bacteremia involved a resistant organism.

\section{Safety Assessment}

The safety population consisted of 113 patients in the levofloxacin group and 118 in the azithromycin group. As seen in 
Table VII. Cross-susceptibility to levofloxacin and ceftriaxone of admission pathogens.

\begin{tabular}{lccccr}
\hline & \multicolumn{5}{c}{ Ceftriaxone, ${ }^{*}$ No. of Isolates } \\
\cline { 2 - 5 } & Susceptible & $\begin{array}{c}\text { Intermediately } \\
\text { Susceptible }\end{array}$ & Resistant & Unknown & Total \\
\hline Levofloxacin, ${ }^{*}$ no. of isolates & & & & & \\
Susceptible & 99 & 3 & 1 & 0 & 103 \\
Intermediately susceptible & 1 & 0 & 0 & 0 & 1 \\
Resistant & 0 & 0 & 0 & 0 & 0 \\
Unknown & 0 & 0 & 0 & 18 & 18 \\
Total & 100 & 3 & 1 & 18 & 122 \\
\hline
\end{tabular}

*Two patients in the levofloxacin group and 3 patients in the comparator group were randomized to treatment but did not receive study medication.

Table VIII. Clinical characteristics and minimum inhibitory concentrations (MICs) of pathogens identified in patients considered treatment failures.

\begin{tabular}{|c|c|c|c|c|}
\hline Treatment/Patient & $\begin{array}{l}\text { Comorbidity/ } \\
\text { Adverse Event }\end{array}$ & $\begin{array}{l}\text { Duration of } \\
\text { Therapy, } \mathrm{d}^{*}\end{array}$ & Pathogen & $\begin{array}{l}\mathrm{MIC}, \\
\mu \mathrm{g} / \mathrm{mL}\end{array}$ \\
\hline
\end{tabular}

Levofloxacin

\begin{tabular}{|c|c|c|c|c|}
\hline 76-year-old woman ${ }^{\dagger}$ & $\mathrm{CHF}$ & 3 & Streptococcus pneumoniae & $\begin{array}{l}\mathrm{L}, 1.0 \\
\mathrm{~A}, 0.12 \\
\mathrm{C}, \leq 0.25\end{array}$ \\
\hline 39-year-old man ${ }^{+}$ & Empyema & 4 & Staphylococcus aureus & $\begin{array}{l}\mathrm{L}, 0.5 \\
\mathrm{~A}, 1.0 \\
\mathrm{C}, 2\end{array}$ \\
\hline 94-year-old $\operatorname{man}^{\dagger \ddagger}$ & Multiorgan failure & 4 & Haemophilus influenzae & NA \\
\hline \multicolumn{5}{|l|}{ Comparator regimen ${ }^{\S}$} \\
\hline 88-year-old man & Respiratory distress & 3 & $H$ influenzae & $\begin{array}{l}\mathrm{L}, 0.5 \\
\mathrm{~A}, 0.5 \\
\mathrm{C}, 0.25\end{array}$ \\
\hline 62-year-old man & $\begin{array}{l}\text { Left testicular } \\
\text { necrosis }\end{array}$ & 5 & Escherichia coli & $\begin{array}{l}\mathrm{L}, 8.0 \\
\mathrm{~A}, 8.0 \\
\mathrm{C}, 0.25\end{array}$ \\
\hline
\end{tabular}

$\mathrm{CHF}=$ congestive heart failure; $\mathrm{L}=$ levofloxacin; $\mathrm{A}=$ azithromycin; $\mathrm{C}=$ ceftriaxone; $\mathrm{NA}=$ not available.

${ }^{*}$ Number of days before discontinuation of study drug.

${ }^{\dagger}$ Microbiologically evaluable patient in whom causative pathogens were not eradicated.

'Patient died of multiorgan failure after 6 days.

'Comparator regimen consisted of azithromycin $500 \mathrm{mg}$ IV q24h for $\geq 2$ days plus ceftriaxone $1 \mathrm{~g} \mathrm{IV} \mathrm{q} 24 \mathrm{~h}$ for

2 days, followed by an optional switch to azithromycin $500 \mathrm{mg} P O \mathrm{q} 24 \mathrm{~h}$ at the investigator's discretion. 
Table IX. Drug-related adverse events.

\begin{tabular}{llc}
\hline & \multicolumn{2}{c}{ No. $(\%)$} \\
\cline { 2 - 3 } & $\begin{array}{c}\text { Levofloxacin } \\
(\mathrm{n}=113)\end{array}$ & $\begin{array}{c}\text { Comparator Regimen } \\
(\mathrm{n}=118)\end{array}$ \\
\hline All body systems & $6(5.3)$ & $11(9.3)$ \\
Gastrointestinal disorders & $0(0)$ & $7(5.9)$ \\
Diarrhea & $0(0)$ & $5(4.2)$ \\
Nausea & $0(0)$ & $1(0.8)$ \\
Pseudomembranous colitis & $0(0)$ & $1(0.8)$ \\
Vascular disorders & $0(0)$ & $3(2.5)$ \\
Vein disorders & $0(0)$ & $3(2.5)$ \\
Skin and appendages & $3(2.7)$ & $2(1.7)$ \\
Pruritus & $0(0)$ & $2(1.7)$ \\
Rash & $1(0.9)$ & $0(0)$ \\
Sweat gland disorder & $1(0.9)$ & $0(0)$ \\
Urticaria & $1(0.9)$ & $0(0)$ \\
Central/peripheral nervous & & \\
system disorders & $1(0.9)$ & $1(0.8)$ \\
Dizziness & $1(0.9)$ & $0(0)$ \\
Hypoesthesia & $0(0)$ & $1(0.8)$ \\
Psychiatric disorders & $2(1.8)$ & $0(0)$ \\
Insomnia & $1(0.9)$ & $0(0)$ \\
Paroniria & $1(0.9)$ & $0(0)$ \\
Body as a whole & $1(0.9)$ & $0(0)$ \\
\hline Comparar & & \\
\hline
\end{tabular}

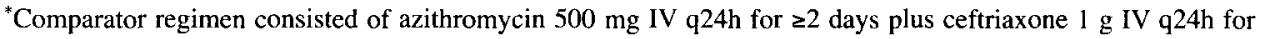
2 days, followed by an optional switch to azithromycin $500 \mathrm{mg}$ PO q24h at the investigator's discretion.

Table IX, the overall incidence of drugrelated AEs for all body systems was $5.3 \%$ in the levofloxacin group and $9.3 \%$ in the azithromycin group. The most common drug-related AEs in the azithromycin group were diarrhea $(4.2 \%)$, vein disorders $(2.5 \%)$, and pruritus $(1.7 \%)$, whereas no drug-related $\mathrm{AE}$ occurred in $\geq 1.0 \%$ of levofloxacin-treated patients. One patient receiving levofloxacin had a serious drugrelated AE, compared with 7 patients receiving azithromycin. Six deaths occurred during the study: 2 in the levofloxacin group and 4 in the azithromycin group. None of the deaths were considered to be treatment related.

\section{DISCUSSION}

Clinical practice guidelines are important tools when choosing treatment for a particular disease state. The IDSA guidelines recommend doxycycline, macrolides, or fluoroquinolones as the preferred antimicrobial agents for the empiric therapy of most outpatient cases of CAP, and either 
a fluoroquinolone alone or a beta-lactam/ beta-lactamase inhibitor or extendedspectrum cephalosporin combined with a macrolide for most hospitalized patients with CAP in general medical wards. ${ }^{9}$ In the present trial, levofloxacin monotherapy was at least as effective and well tolerated as traditional combination therapy with a macrolide and a beta-lactam in the treatment of moderate to severe CAP. The pharmacokinetics of levofloxacin are such that the drug achieves high concentrations soon after dosing that are sustained at levels far exceeding the MIC required to achieve $90 \%$ inhibition of most respiratory pathogens in both serum and lung tissue. ${ }^{17}$ Reports suggest that levofloxacin is effective in the treatment of pneumococcal CAP (including bacteremic infections) in patients who have failed macrolide therapy, as well as patients with bacteremic CAP. ${ }^{18,19}$ Most patients in the present study had significant disease, as indicated by a mean Fine risk score of 93.4. They were randomized to once-daily treatment with levofloxacin IV or PO or $\geq 2$ days of combination therapy with azithromycin and ceftriaxone IV, followed by an optional transition to azithromycin PO, for a total duration of treatment of $\geq 10$ days. The addition of ceftriaxone to the initial azithromycin regimen was considered a necessary safeguard in patients with pneumococcal bacteremia. However, because azithromycin was not used as monotherapy, this made it difficult to evaluate the clinical and microbiologic outcome with azithromycin alone.

The results of this trial suggest that levofloxacin is at least as effective as azithromycin, despite the fact that azithromycin was initially coadministered with ceftriaxone. Some of the inclusion and exclusion criteria may have limited the ability to extrapolate from the results. The clinical success rate in clinically evaluable patients was $94.1 \%$ in the levofloxacin group and $92.3 \%$ in the azithromycin group. The respective microbiologic eradication rates were $89.5 \%$ and $92.3 \%$. A strong correlation was observed between the clinical and microbiologic response in both treatment groups. Twelve patients in the levofloxacin group received oral levofloxacin only, and the clinical success rate in this group was $100 \%$. A total of 5 patients were classified as treatment failures, all of whom had received study drug for $\leq 5$ days and all with significant comorbidity.

Levofloxacin was as well tolerated as azithromycin, with an overall incidence of AEs related to all body systems of $5.3 \%$ and $9.3 \%$ of patients, respectively. The most common drug-related AEs associated with azithromycin therapy were diarrhea, vein disorders, and pruritus, whereas no drug-related $\mathrm{AE}$ occurred in $>1.0 \%$ of levofloxacin-treated patients. Although 6 deaths occurred during the study, none were considered treatment related.

\section{CONCLUSIONS}

Based on its clinical and microbiologic efficacy, as well as its favorable tolerability profile, levofloxacin monotherapy appears to be effective in the management of hospitalized patients with moderate to severe CAP.

\section{ACKNOWLEDGMENTS}

The authors thank the following for their contributions to this study: Igor Abolnik, MD, Salt Lake City Veterans Administration Hospital, Salt Lake City, Utah; Kenneth C. Anderson, MD, Baptist East Hos- 
pital, Louisville, Kentucky; Marvin J. Bittner, MD, Omaha Veterans Administration Medical Center, Omaha, Nebraska; Robert Owen Brennan, MD, Medical Associates of Central Virginia, Lynchburg, Virginia; Gary Decker, MD, Infectious Disease Associates, Wilkes-Barre, Pennsylvania; M. Pia DeGirolamo, MD, Grandview Hospital, Sellersville, Pennsylvania; Elliott Frank, MD, Jersey Shore Medical Center, Neptune, New Jersey; Gary Kinasewitz, MD, University of Oklahoma Health Sciences Center, Oklahoma City, Oklahoma; Howard M. Lazarus, MD, Harrison Memorial Hospital, Bremerton, Washington; Jerome F. Levine, MD, Hackensack University Medical Center, Hackensack, New Jersey; Diane K. Litwin, MD, St. Elizabeth Medical Center, Edgewood, Kentucky; Jing Liu, MD, Salem Veterans Affairs Medical Center, Salem, Virginia; Christopher Lucasti, DO, South Jersey Infectious Disease, Somers Point, New Jersey; Gregory Moran, MD, Olive View-UCLA Medical Center, Sylmar, California; Joseph Plouffe, MD, Ohio State University Medical Center, Columbus, Ohio; Annette Rebouli, MD, Cooper Hospital University Medical Center, Camden, New Jersey; Paul Scheinberg, MD, Saint Joseph's Hospital of Atlanta, Atlanta, Georgia; Leon Smith, MD, St. Michael Medical Center, Newark, New Jersey; and Marcus Zervos, MD, William Beaumont Hospital, Royal Oak, Michigan.

\section{REFERENCES}

1. Ginesu F, Pirina P, Deiola G, et al. Etiology and therapy of community-acquired pneumonia. J Chemother. 1997:9:285-292.

2. Ewig S, Ruiz M, Mensa J, et al. Severe community-acquired pneumonia. Assess- ment of severity criteria. Am J Respir Crit Care Med. 1998;158:1102-1108.

3. Marrie TJ. Community-acquired pneumonia: Epidemiology, etiology, treatment. $I n$ fect Dis Clin North Am. 1998;12:723-740.

4. Ewig S, Torres A. Severe communityacquired pneumococcal pneumoniawhat might be done better. Intensive Care Med. 1999;25:143-145.

5. Reynolds HY. Respiratory infections: Community-acquired pneumonia and newer microbes. Lung. 1996;174:207-224.

6. So HY. Severe community-acquired pneumonia. Anaesth Intensive Care. 1997;25: 222-234.

7. Waterer GW, Somes GW, Wunderink RG. Monotherapy may be suboptimal for severe bacteremic pneumococcal pneumonia. Arch Intern Med. 2001;161:1837-1842.

8. Kahn JB, Wiesinger BA, Drucker LM, et al. Cumulative clinical trial experience with levofloxacin in community acquired pneumonia-associated pneumococcal bacteremia. Abstract and poster presented at: The Sixth International Conference on the Macrolides, Azalides, Streptogramins, Ketolides and Oxazolidinones; January 23-26, 2002; Bologna, Italy. Abstract 8.21.

9. Bartlett JG, Dowell SF, Mandell LA, et al, for the Infectious Diseases Society of America. Practice guidelines for the managcment of community-acquired pneumonia in adults. Clin Infect Dis. 2000;31: 347-382.

10. Lonks JR, Garau J, Gomez L, et al. Failure of macrolide treatment of erythromycinresistant Streptococcus pneumoniae. Abstract and poster presented at: The Sixth International Conference on the Macrolides, Azalides, Streptogramins, Ketolides 
and Oxazolidinones; January 23-26, 2002; Bologna, Italy. Abstract 8.36.

11. Fogarty C, Goldschmidt R, Bush K. Bacteremic pneumonia duc to multidrugresistant pneumococci in 3 patients treated unsuccessfully with azithromycin and successfully with levofloxacin. Clin Infect Dis. 2000;31:613-615.

12. Fine MJ, Smith MA, Carson CA, et al. Prognosis and outcomes of patients with community-acquired pneumonia. A metaanalysis. JAMA. 1996;275:134-141.

13. Levaquin ${ }^{\circledR}$ prescribing information. Rari$\tan , \mathrm{NJ}$ : Ortho-McNeil Pharmaceutical; 2002.

14. Zithromax ${ }^{(B)}$ prescribing information. New York: Pfizer Inc; 2002.

15. Hauck WW, Anderson S. A comparison of large-sample confidence interval methods for the difference of two binomial probabilities. Am Statistician. 1986;40:318-322.

16. Guidance for Industry-Draft. Developing Antimicrobial Drugs-General Considerations for Clinical Trials. July 1998. Available at: http://www.fda.gov/cder/guidance/ 2580dft.pdf. Accessed May 8, 2002.

17. Gotfried MH, Danziger LH, Rodvold KA. Steady-state plasma and intrapulmonary concentrations of levofloxacin and ciprofloxacin in healthy adult subjects. Chest. 2001;119:1114-1122.

18. File TM Jr. Levofloxacin in the treatment of community acquired pneumonia. C'an Respir J. 1999;6(Suppl A):35A-39A.

19. Fogarty CM, Sullivan JG, Chattman MS, et al. Once a day levofloxacin in the treatment of mild to moderate and severe communityacquired pneumonia in adults. Infect Dis Clin Pract. 1998;7:400-407.

Address correspondence to: James B. Kahn, MD, Ortho-McNeil Pharmaceutical, 1000 Route 202 South, Raritan, New Jersey 08869-0602. 